\title{
Tanshinone IIA can inhibit MiaPaCa-2 human pancreatic cancer cells by dual blockade of the Ras/Raf/MEK/ERK and PI3K/AKT/mTOR pathways
}

\author{
CHIN-CHENG SU ${ }^{1-4}$ \\ ${ }^{1}$ Tumor Research Center of Integrative Medicine; ${ }^{2}$ Comprehensive Breast Cancer Center; \\ ${ }^{3}$ Department of Surgery, Changhua Christian Hospital, Changhua 50006; ${ }^{4}$ School of Chinese Medicine, \\ College of Chinese Medicine, China Medical University, Taichung 40402, Taiwan, R.O.C.
}

Received January 19, 2018; Accepted August 14, 2018

DOI: $10.3892 /$ or.2018.6670

\begin{abstract}
Tanshinone IIA (Tan-IIA; $\mathrm{C}_{19} \mathrm{H}_{18} \mathrm{O}_{3}$ ) is derived from Danshen (the roots of Salvia miltiorrhiza), and has been reported to possess anti-inflammatory and antioxidant activities. Tan-IIA can inhibit BxPC-3 human pancreatic cancer cells in vitro through inducing endoplasmic reticulum stress and apoptosis via mitochondrial pathways. However, the efficacy and molecular mechanisms of Tan-IIA in human pancreatic cancer have not yet been elucidated. The transmembrane tyrosine kinases, including insulin-like growth factor 1 receptor (IGF1R), vascular endothelial growth factor receptor (VEGFR) or epidermal growth factor receptor (EGFR) have been implicated in the survival and metastasis of cancer. In addition, the Ras/Raf/mitogen-activated protein kinase kinase (MEK)/extracellular signal-regulated kinase (ERK) and phosphoinositide 3-kinase (PI3K)/protein kinase B (AKT)/mammalian target of rapamycin (mTOR) pathways are the most commonly dysregulated kinase cascades in human cancer. The present study aimed to investigate the efficacy and molecular mechanisms of Tan-IIA in MiaPaCa-2 human pancreatic carcinoma cells. The protein expression levels of EGFR, IGF1R, VEGFR, Ras, PI3K, AKT, mTOR, Raf, MEK, ERK and phosphatase and tensin homolog (PTEN) were detected in Tan-IIA-treated MiaPaCa-2 cells by western blotting. The results demonstrated that the protein expression levels of EGFR, IGF1R, VEGFR, Ras, Raf, MEK, ERK, PI3K, AKT, mTOR and PTEN were decreased in MiaPaCa-2 cells treated with various concentrations of Tan-IIA for different durations. In conclusion, these findings indicated that Tan-IIA may inhibit MiaPaCa-2 human pancreatic cancer cells; the molecular mechanisms underlying this inhibitory effect may
\end{abstract}

Correspondence to: Dr Chin-Cheng Su, Tumor Research Center of Integrative Medicine, Changhua Christian Hospital, 135 Nan-Hsiao Street, Changhua 50006, Taiwan, R.O.C.

E-mail: succ.maeva@msa.hinet.net

Key words: Tan-IIA, MiaPaCa-2 cells, Ras, Raf, PI3K be involved in downregulating EGFR, IGF1R and VEGFR expression, and dual blockade of the Ras/Raf/MERK/ERK and PI3K/AKT/mTOR pathways.

\section{Introduction}

Pancreatic cancer was revealed to be the fourth leading cause of cancer-associated mortality in the USA in 2016, and the estimated number of deaths caused by pancreatic cancer for that year is 21,450 in men and 20,330 in women (1). Numerous efforts and chemotherapeutic advancements have been made to improve the efficacy of pancreatic cancer treatment; however, the results remain unsatisfactory (2-4). Therefore, there is an urgent need to develop novel therapeutic agents for pancreatic cancer.

It is well documented that transmembrane tyrosine kinases are strongly associated with the proliferation and metastasis of numerous types of human cancer $(5,6)$. The phosphoinositide 3-kinase $(\mathrm{PI} 3 \mathrm{~K}) /$ protein kinase $\mathrm{B}$ $(\mathrm{AKT}) / \mathrm{mammalian}$ target of rapamycin (mTOR) and $\mathrm{Ras} / \mathrm{Raf} / \mathrm{mitogen}$-activated protein kinase kinase (MEK)/ extracellular signal-regulated kinase (ERK) pathways are two of the most frequently dysregulated kinase cascades in human cancer. These pathways signify important signal transduction mechanisms, which accelerate the proliferation and survival of cancers via activation of growth factor receptors, such as insulin-like growth factor 1 receptor (IGF1R), vascular endothelial growth factor receptor (VEGFR) and epidermal growth factor receptor (EGFR) (7,8). Either through somatic mutations or epigenetic modifications, the individual downstream constituents of these signaling cascades can induce tumorigenesis and resistance to anticancer treatments, and these constituents have been reported to be frequently transformed in malignant tumors (9).

Tanshinone-IIA (Tan-IIA) is an active component of the plant-derived traditional Chinese medicine Danshen, which has been reported to possess anticancer potential $(2,3)$. Tan-IIA is able to inhibit the protein expression levels of MCL1, B-cell lymphoma 2 (Bcl-2) family apoptosis regulator; tumor protein, translationally-controlled 1 and Bcl-extra large, in order to destroy mitochondrial function and increase Bcl-2-associated 
$\mathrm{X}$ protein and caspase-3 expression, thus inducing apoptosis of BxPC-3 human pancreatic cancer cells (10). Furthermore, Tan-IIA increases protein kinase R-like endoplasmic reticulum kinase, activating transcription factor 6 , caspase-12 and CCAAT-enhancer-binding protein homologous protein expression, in order to induce apoptosis of $\mathrm{BxPC}-3$ pancreatic cancer cells in vitro (11). It has also been reported that Tan-IIA may exert cytotoxic effects on $\mathrm{MiaPaCa}-2$ human pancreatic cancer cells (12). The present study aimed to detect the protein expression levels of IGF1R, VEGFR, EGFR, PI3K, AKT, mTOR, Ras, Raf, MEK, ERK and phosphatase and tensin homolog (PTEN) in MiaPaCa-2 human pancreatic cancer cells following treatment with Tan-IIA.

\section{Materials and methods}

Chemicals and reagents. Tan-IIA (molecular formula, $\mathrm{C}_{19} \mathrm{H}_{18} \mathrm{O}_{3}$; CAS No., 568-72-9) was purchased from Sigma-Aldrich; Merck KGaA (Darmstadt, Germany). The MiaPaCa-2 human pancreatic cancer cell line (BCRC No. 60139) was obtained from the Food Industry Research and Development Institute (Hsinchu, Taiwan). MTT, sodium deoxycholate, leupeptin, Triton X-100, Tris/HCl, RNase A, sodium orthovanadate, sodium pyruvate, HEPES and mouse anti- $\beta$-actin (cat no. A5441, MW 43 kDa) were obtained from Sigma-Aldrich; Merck KGaA. Dimethyl sulfoxide (DMSO), potassium phosphate and TE buffer were purchased from Merck KGaA. Fetal bovine serum (FBS), Dulbecco's modified Eagle's medium (DMEM), trypsin-EDTA, penicillin-streptomycin and glutamine were obtained from Gibco; Thermo Fisher Scientific, Inc. (Waltham, MA, USA). Buffer (10X TG-SDS), Tween-20 and glycine were obtained from Amresco, LLC (Solon, OH, USA). BioMax film was obtained from Kodak (Rochester, NY, USA). Other materials and reagents not specified were obtained from Sigma-Aldrich; Merck KGaA or Merck KGaA.

Antibodies. Anti-VEGFR (cat. no. NB100-527, MW 151 kDa) was obtained from Novus Biologicals, LLC (Littleton, CO, USA); anti-EGFR (cat. no. 2239, MW 175 kDa), anti-IGF1R (cat. no. 3018, MW 95 kDa), anti-Ras (cat. no. 3339, MW $21 \mathrm{kDa}$ ), anti-Raf (cat. no. 12552, MW 75 kDa), anti-MEK (cat. no. 9126, MW 45 kDa), anti-ERK (cat. no. 4695, MW 42-44 kDa), anti-PI3K (cat. no. 4292, MW 85 kDa), anti-AKT (cat. no. 3063, MW 60 kDa), anti-mTOR (cat. no. 2983, MW $289 \mathrm{kDa}$ ) and anti-PTEN cat. no. 9559, MW $54 \mathrm{kDa}$ ) antibodies were all obtained from Cell Signaling Technology, Inc. (Danvers, MA, USA).

Cell culture. The MiaPaCa-2 cells were maintained in DMEM containing $10 \% \mathrm{FBS}$ and $1 \%$ penicillin/streptomycin (10,000 U/ml penicillin, $10 \mathrm{mg} / \mathrm{ml}$ streptomycin) at $37^{\circ} \mathrm{C}$ in a humidified atmosphere containing $5 \% \mathrm{CO}_{2}$.

Cytotoxicity assay. The cytotoxicity of Tan-IIA in MiaPaCa-2 cells was evaluated by MTT assay in triplicate, as previously described (10). Briefly, the MiaPaCa- 2 cells were plated in 96-well plates at a density of $1 \times 10^{4}$ cells/well and were treated with various concentrations $(0,1,3,9,15,30$ and $60 \mu \mathrm{g} / \mathrm{ml})$ of Tan-IIA for different durations (24, 48 and $72 \mathrm{~h})$. Subsequently, the cells were incubated with $100 \mu \mathrm{l}$ MTT $(1 \mathrm{mg} / \mathrm{ml})$ in fresh complete DMEM for $2 \mathrm{~h}$. The surviving cells converted MTT to formazan, as presented by a blue-purple color when dissolved in DMSO at $37^{\circ} \mathrm{C}$ for $2 \mathrm{~h}$. Absorbance was measured using an ELISA microplate reader at $590 \mathrm{~nm}$. The relative percentage of cell viability was calculated by dividing the absorbance of treated cells by that of the control in each experiment, using the following formula: Proliferation rate $(\%)=(O D$ test - OD blank) x 100, where OD test and OD blank are the optical density of the test substance and the blank control, respectively.

Western blotting. The effects of Tan-IIA on the protein expression levels of EGFR, IGF1R, VEGFR, Ras, PI3K, AKT, mTOR, Raf, MEK, ERK and PTEN were detected in MiaPaCa- 2 cells. The MiaPaCa- 2 cells were treated with various concentrations of Tan-IIA $(0,3,6$ and $12 \mu \mathrm{g} / \mathrm{ml})$ for $48 \mathrm{~h}$, or with various concentrations of Tan-IIA $(0,1.5,3$ and $6 \mu \mathrm{g} / \mathrm{ml})$ for $72 \mathrm{~h}$, after which the expression levels of EGFR, IGF1R, VEGFR, Ras, PI3K, AKT, mTOR, Raf, MEK, ERK and PTEN were evaluated by western blotting. Alternatively, MiaPaCa-2 cells were treated with Tan-IIA $(6 \mu \mathrm{g} / \mathrm{ml})$ for various durations $(0,24,48$ and $72 \mathrm{~h})$, after which the proteins expression levels of EGFR, IGF1R, VEGFR, Ras, PI3K, AKT, mTOR, Raf, MEK, ERK and PTEN were evaluated by western blotting.

Western blot analysis was conducted, as previously described (10). Briefly, after treatment, the cells were lysed in ice-cold whole cell extract buffer containing protease inhibitors (cat. no. 20-188; Merck KGaA). The lysate was agitated for $30 \mathrm{~min}$ at $4^{\circ} \mathrm{C}$ and centrifuged at $12,281 \mathrm{x} \mathrm{g}$ for $10 \mathrm{~min}$. Protein concentration was measured using a bicinchoninic acid protein assay kit (Pierce; Thermo Fisher Scientific, Inc.). Equal amounts of protein $(10 \mu \mathrm{g})$ were then subjected to electrophoresis using 10-15\% SDS-polyacrylamide gels. To verify equal protein loading $(10 \mu \mathrm{g})$ and transfer, proteins were transferred to polyvinylidene difluoride membranes, which were blocked overnight at $4{ }^{\circ} \mathrm{C}$ using blocking buffer [5\% non-fat dried milk in solution containing $50 \mathrm{mM}$ Tris/ $\mathrm{HCl}$ (pH 8.0), $2 \mathrm{mM}$ $\mathrm{CaCl}_{2}, 80 \mathrm{mM}$ sodium chloride, $0.05 \%$ Tween-20 and $0.02 \%$ sodium azide]. The membranes were then incubated with the specific primary antibodies $(1: 1,000)$ for $2 \mathrm{~h}$ at $25^{\circ} \mathrm{C}$ and were washed three times with Tris-buffered saline- $0.05 \%$ Tween-20 (TBST). Subsequently, the membranes were incubated with anti-rabbit (cat. no. sc-2004) or anti-mouse (cat. no. sc-2005) immunoglobulin G-horseradish peroxidase-conjugated secondary antibodies (1:5,000; Santa Cruz Biotechnology Inc., Dallas, TX, USA). The membranes were then washed a further three times for $10 \mathrm{~min}$ with TBST. Finally, the protein bands were visualized on X-ray film and were analyzed using the enhanced chemiluminescence detection system (PerkinElmer, Inc., Waltham, MA, USA) and ImageJ 1.4.4p (National Institutes of Health, Bethesda, MD, USA) software analysis. $\beta$-actin was used as an internal control in all western blots. Results are presented as the means \pm standard deviation of three experiments.

Statisticalanalysis. The statistical significance of the differences between groups was assessed using SPSS software version 20 (IBM Corp., Armonk, NY, USA). Data were analyzed using one-way analysis of variance followed by Dunnett's test. $\mathrm{P}<0.05$ was considered to indicate a statistically significant difference. 


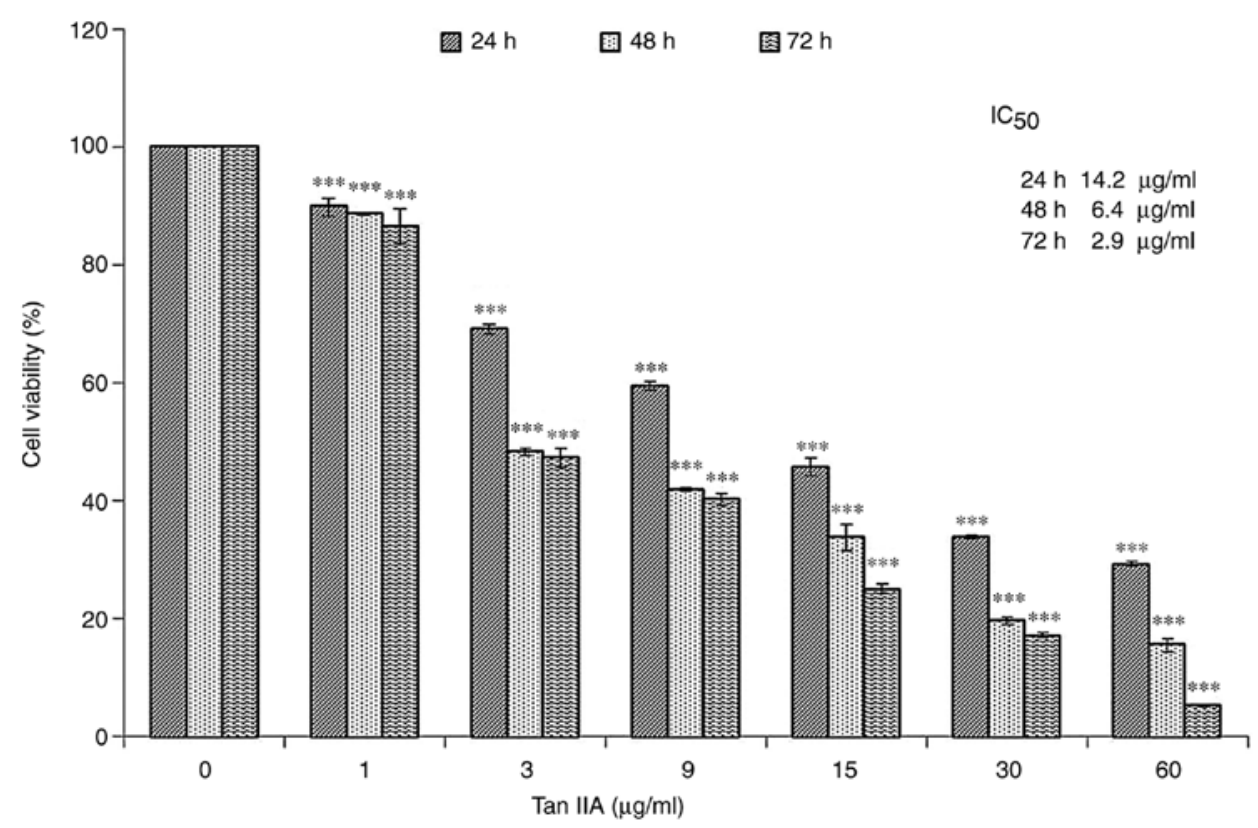

Figure 1. Cytotoxicity of Tan-IIA in MiaPaCa-2 cells. Cytotoxicity of Tan-IIA in MiaPaCa-2 cells was determined using the MTT assay. MiaPaCa-2 cells were cultured with various concentrations $(0,1,3,9,15,30$ and $60 \mu \mathrm{g} / \mathrm{ml})$ of Tan-IIA for different durations $(24,48$ and $72 \mathrm{~h})$. The results revealed that Tan-IIA inhibited the proliferation of MiaPaCa-2 human pancreatic cancer cells in a time- and dose-dependent manner. The $\mathrm{IC}_{50}$ values were $14.2,6.4 \mathrm{and} 2.9 \mu \mathrm{g} / \mathrm{ml}$, respectively. Results are presented as the means \pm standard deviation of three experiments. ${ }^{* * * *} \mathrm{P}<0.001$ vs. the control group. $\mathrm{IC}_{50}$, half-maximum inhibitory concentration; Tan-IIA, Tanshinone-IIA.
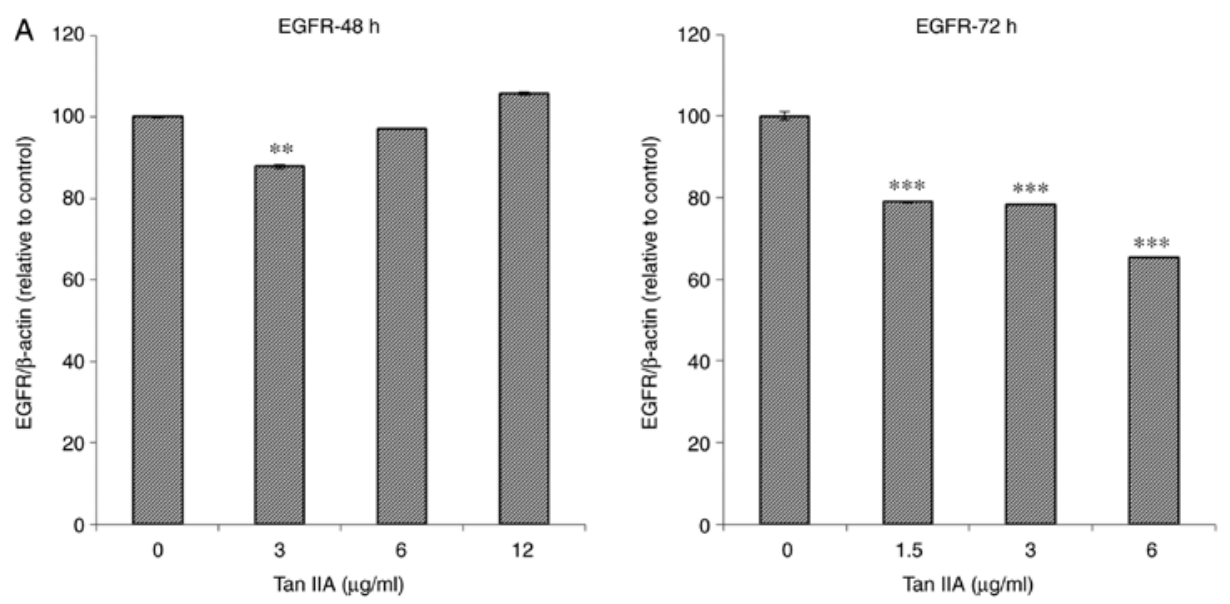

Figure 2. Dose-dependent effects of Tan-IIA on the protein expression levels of EGFR, IGF1R, VEGFR, Ras, Raf, MEK, ERK,PI3K, AKT, mTOR and PTEN in MiaPaCa-2 cells. The MiaPaCa-2 cells were treated with various concentrations $(0,3,6$ and $12 \mu \mathrm{g} / \mathrm{ml})$ of Tan-IIA for $48 \mathrm{~h}$ or with various concentrations $(0,1.5,3$ and $6 \mu \mathrm{g} / \mathrm{ml})$ of Tan-IIA for $72 \mathrm{~h}$, and the protein expression levels were evaluated by western blot analysis. Protein expression levels of (A) EGFR. ${ }^{* *} \mathrm{P}<0.01,{ }^{* * *} \mathrm{P}<0.001$ compared with the control group. AKT, protein kinase B; EGFR, epidermal growth factor receptor; ERK, extracellular signal-regulated kinase; IGF1R, insulin-like growth factor 1 receptor; MEK, mitogen-activated protein kinase kinase; mTOR, mammalian target of rapamycin; PI3K, phosphoinositide 3-kinase; PTEN, phosphatase and tensin homolog; VEGFR, vascular endothelial growth factor receptor.

\section{Results}

Cytotoxicity of Tan-IIA in MiaPaCa-2 cells. MiaPaCa-2 cells were cultured with various concentrations $(0,1,3,9,15,30$ and $60 \mu \mathrm{g} / \mathrm{ml})$ of Tan-IIA for different durations $(24,48$ and $72 \mathrm{~h})$. Following Tan-IIA treatment for 24,48 and $72 \mathrm{~h}$, the half maximum inhibitory concentration values for Tan-IIA were 14.2, 6.4 and $2.9 \mu \mathrm{g} / \mathrm{ml}$, respectively. These results revealed that Tan-IIA may inhibit the proliferation of MiaPaCa-2 human pancreatic cancer cells in a time- and dose-dependent manner (Fig. 1).
Dose-dependent effects of Tan-IIA on the protein expression levels of EGFR, IGF1R, VEGFR, Ras, Raf, MEK, ERK, PI3K, AKT, mTOR and PTEN in MiaPaCa-2 cells. MiaPaCa-2 cells were treated with various concentrations $(0,3,6$ and $12 \mu \mathrm{g} / \mathrm{ml})$ of Tan-IIA for $48 \mathrm{~h}$ or with various concentrations $(0,1.5,3$ and $6 \mu \mathrm{g} / \mathrm{ml}$ ) of Tan-IIA for $72 \mathrm{~h}$, after which the protein expression levels were evaluated by western blot analysis (Fig. 2). The results revealed that Tan-IIA significantly decreased the protein expression levels of Ras (Fig. 2D and M), Raf (Fig. 2E and L), MEK (Fig. 2F and M),ERK (Fig. 2G and M),AKT (Fig. 2I and L) and PTEN (Fig. 2K and L) at 48 and $72 \mathrm{~h}$. However, the 

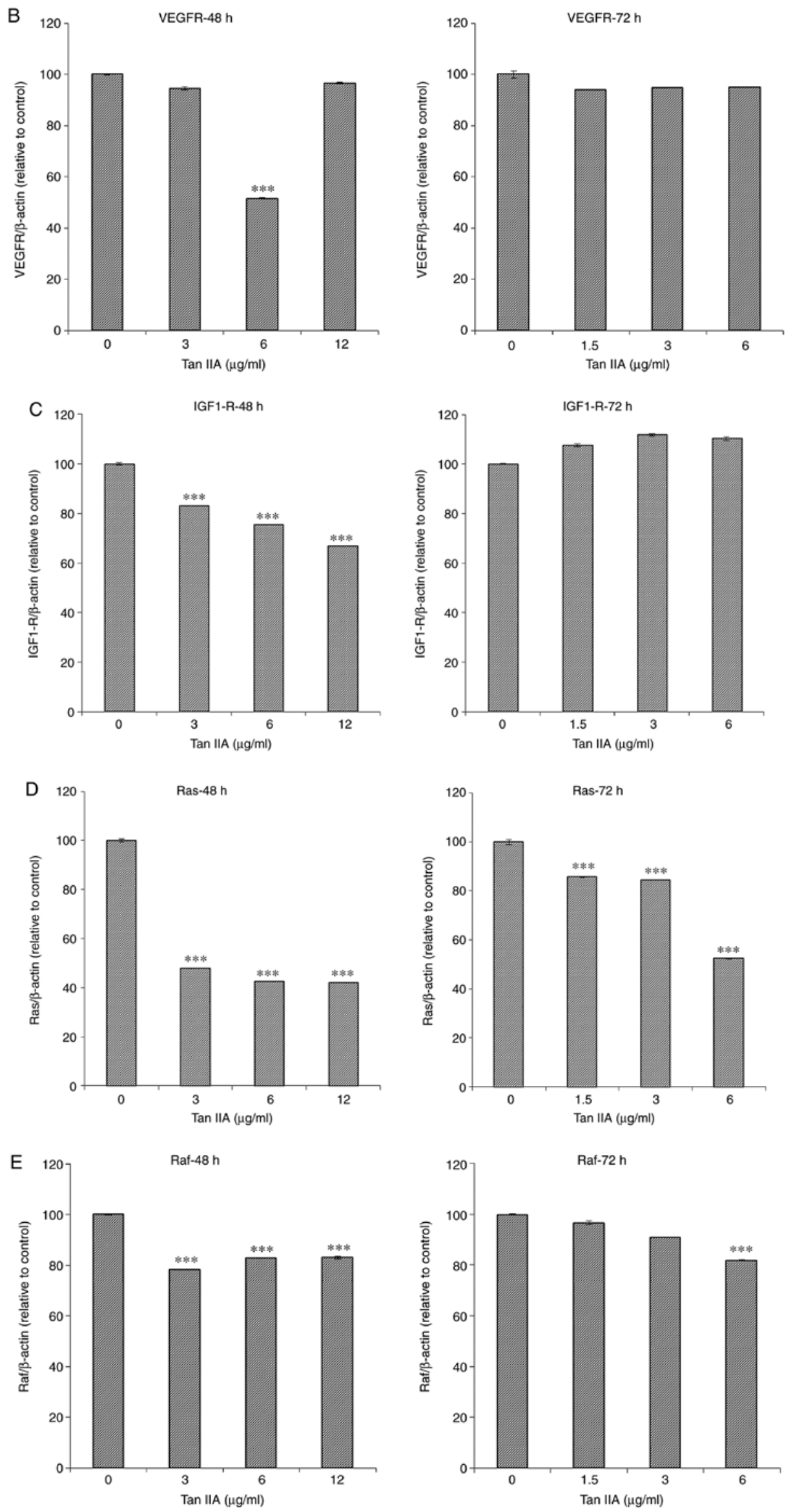

Figure 2. Continued. Protein expression levels of (B) VEGFR, (C) IGF1R, (D) Ras, (E) Raf. ${ }^{* * *}$ P $<0.001$ compared with the control group. AKT, protein kinase B; EGFR, epidermal growth factor receptor; ERK, extracellular signal-regulated kinase; IGF1R, insulin-like growth factor 1 receptor; MEK, mitogen-activated protein kinase kinase; mTOR, mammalian target of rapamycin; PI3K, phosphoinositide 3-kinase; PTEN, phosphatase and tensin homolog; VEGFR, vascular endothelial growth factor receptor. 

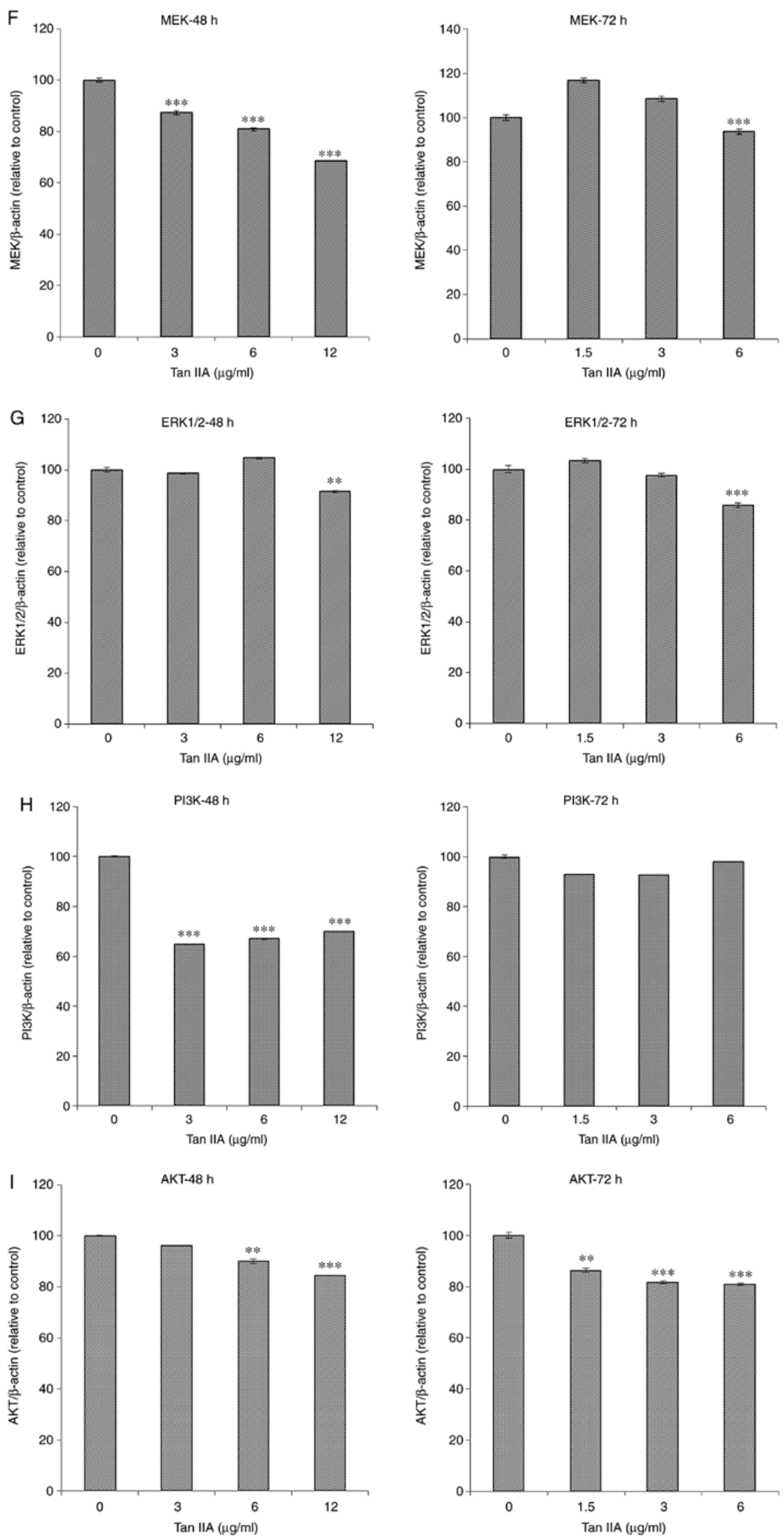

Figure 2. Continued. Protein expression levels of (F) MEK, (G) ERK, (H) PI3K, (I) AKT ${ }^{* *} \mathrm{P}<0.01,{ }^{* * *} \mathrm{P}<0.001$ compared with the control group. AKT, protein kinase B; EGFR, epidermal growth factor receptor; ERK, extracellular signal-regulated kinase; IGF1R, insulin-like growth factor 1 receptor; MEK, mitogen-activated protein kinase kinase; mTOR, mammalian target of rapamycin; PI3K, phosphoinositide 3-kinase; PTEN, phosphatase and tensin homolog; VEGFR, vascular endothelial growth factor receptor. 

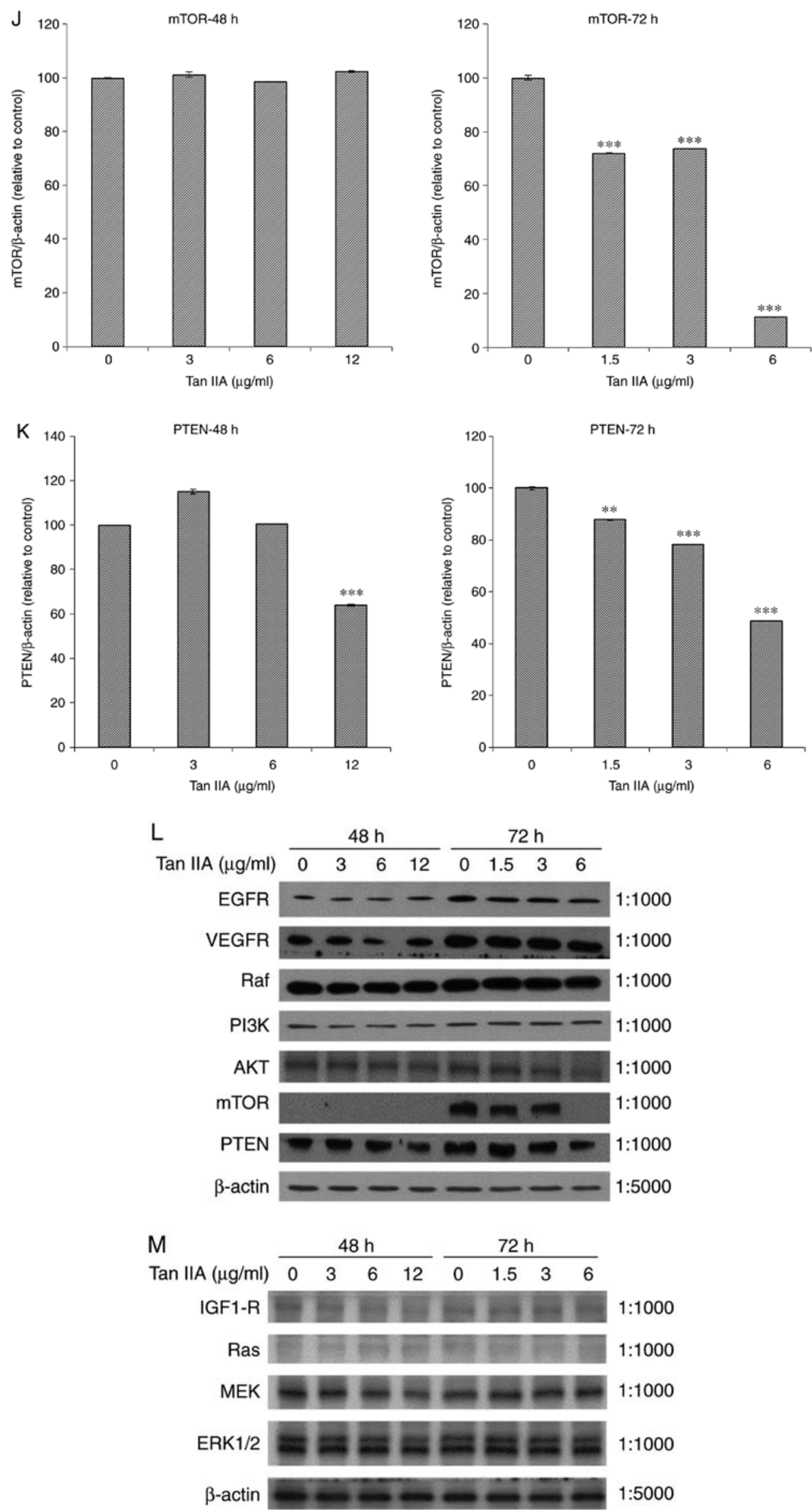

Figure 2. Continued. (J) mTOR and (K) PTEN. (L and M) Representative images of western blots. ${ }^{* *} \mathrm{P}<0.01,{ }^{* * * *} \mathrm{P}<0.001$ compared with the control group. AKT, protein kinase B; EGFR, epidermal growth factor receptor; ERK, extracellular signal-regulated kinase; IGF1R, insulin-like growth factor 1 receptor; MEK, mitogen-activated protein kinase kinase; mTOR, mammalian target of rapamycin; PI3K, phosphoinositide 3-kinase; PTEN, phosphatase and tensin homolog; VEGFR, vascular endothelial growth factor receptor. 

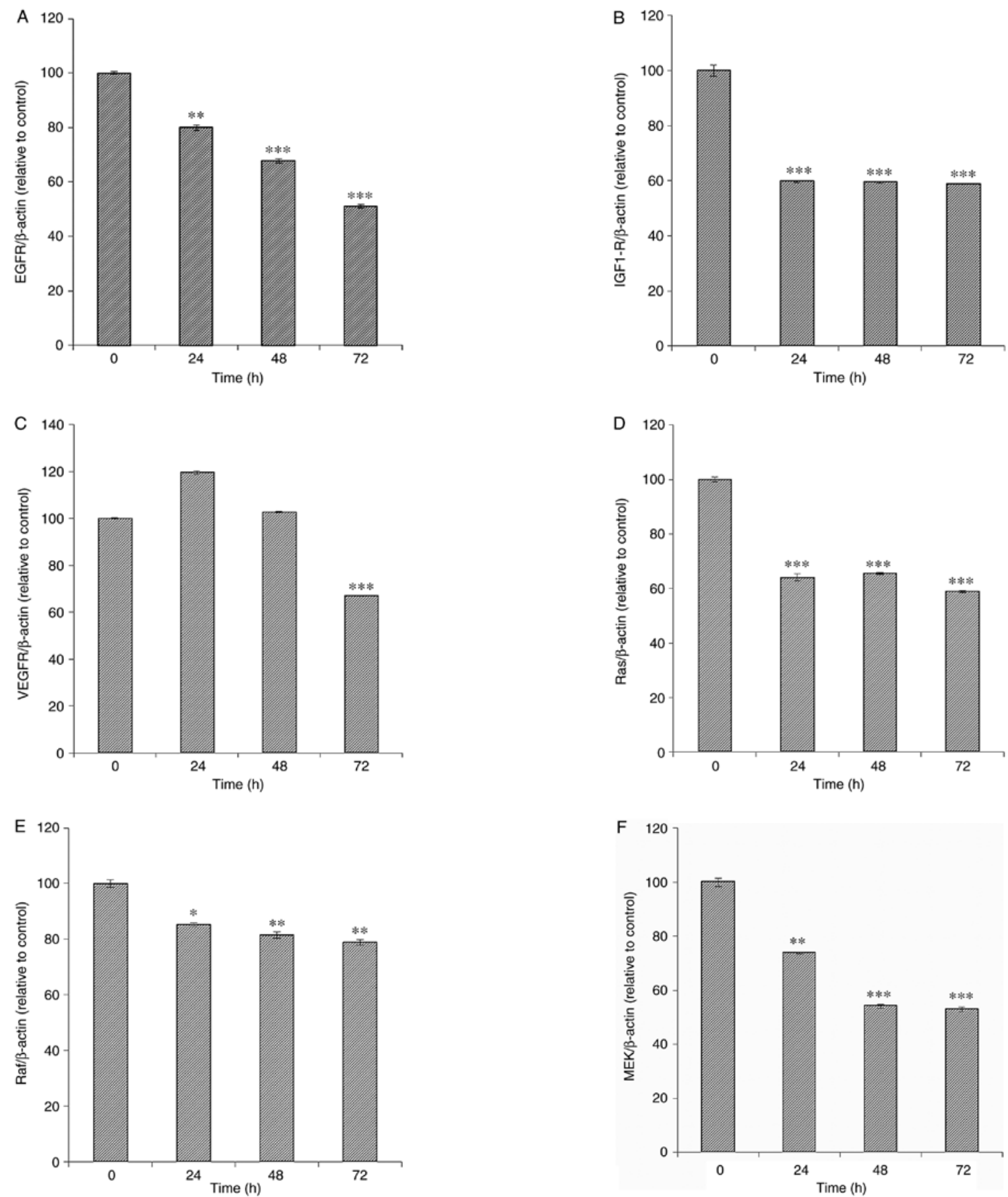

Figure 3. Time-dependent effects of Tan-IIA on the protein expression levels of EGFR, IGF1R, VEGFR, Ras, Raf, MEK, ERK,PI3K, AKT, mTOR and PTEN in MiaPaCa-2 cells. MiaPaCa- 2 cells were treated with Tan-IIA $(6 \mu \mathrm{g} / \mathrm{ml})$ for various durations $(0,24,48$ and $72 \mathrm{~h})$ and the protein expression levels were evaluated by western blotting. Protein expression levels of (A) EGFR, (B) IGF1R, (C) VEGFR, (D) Ras, (E) Raf, (F) MEK "P<0.05, ${ }^{* * *} \mathrm{P}<0.01,{ }^{* * * *} \mathrm{P}<0.001$ compared with the control group. AKT, protein kinase B; EGFR, epidermal growth factor receptor; ERK, extracellular signal-regulated kinase; IGF1R, insulin-like growth factor 1 receptor; MEK, mitogen-activated protein kinase kinase; mTOR, mammalian target of rapamycin; PI3K, phosphoinositide 3-kinase; PTEN, phosphatase and tensin homolog; VEGFR, vascular endothelial growth factor receptor.

protein expression levels of IGF1R (Fig. 2C and M) and PI3K (Fig. 2H and L) were significantly decreased only in response to $48 \mathrm{~h}$ treatment. The protein expression levels of mTOR (Fig. 2J and L) were significantly decreased only in response to $72 \mathrm{~h}$ treatment. The protein expression levels of EGFR (Fig. 2A and L) were significantly decreased in response to all concentrations of Tan-IIA after $72 \mathrm{~h}$ and in response to $3 \mu \mathrm{g} / \mathrm{ml}$ Tan-IIA after $48 \mathrm{~h}$.
Time-dependent effects of Tan-IIA on the protein expression levels of EGFR, IGFIR, VEGFR, Ras, Raf, MEK, ERK,PI3K, $A K T$, mTOR and PTEN in MiaPaCa-2 cells. MiaPaCa-2 cells were treated with Tan-IIA $(6 \mu \mathrm{g} / \mathrm{ml})$ for various durations $(0,24,48$ and $72 \mathrm{~h})$ and the protein expression levels were evaluated by western blot analysis (Fig. 3). The results revealed that Tan-IIA significantly decreased the protein expression levels of EGFR (Fig. 3A and L), IGF1R (Fig. 3B and L), 

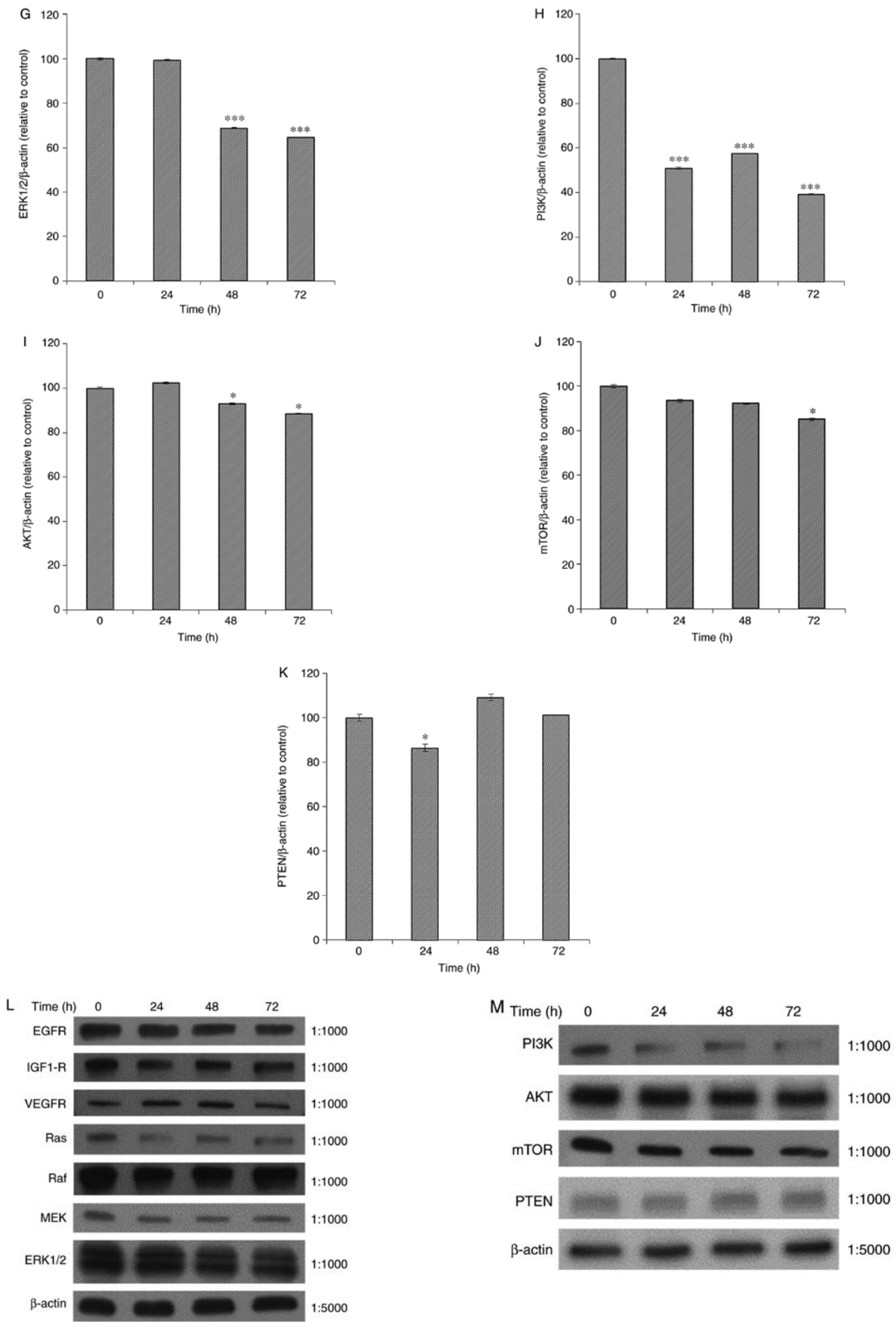

Figure 3. Continued. Protein expression levels of (G) ERK, (H) PI3K, (I) AKT, (J) mTOR and (K) PTEN. (L and M) Representative images of western blots. ${ }^{*} \mathrm{P}<0.05,{ }^{* * * *} \mathrm{P}<0.001$ compared with the control group. AKT, protein kinase B; EGFR, epidermal growth factor receptor; ERK, extracellular signal-regulated kinase; IGF1R, insulin-like growth factor 1 receptor; MEK, mitogen-activated protein kinase kinase; mTOR, mammalian target of rapamycin; PI3K, phosphoinositide 3-kinase; PTEN, phosphatase and tensin homolog; VEGFR, vascular endothelial growth factor receptor. 
Tan IIA

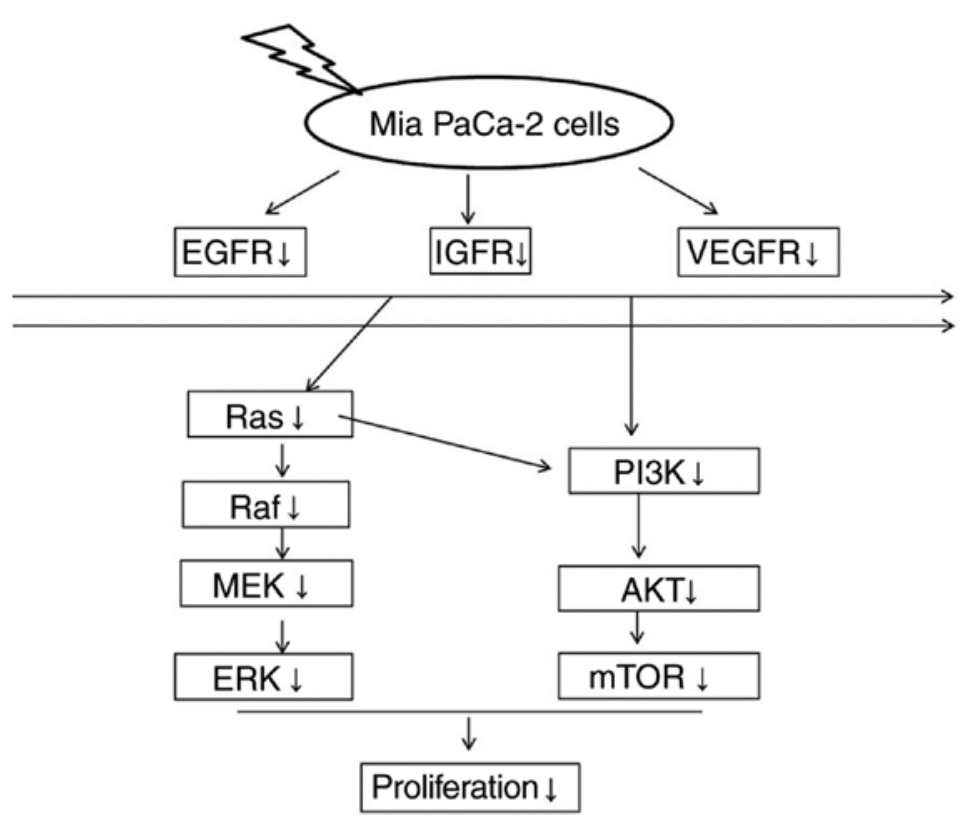

Figure 4. Proposed model for the molecular mechanisms underlying the inhibitory effects of Tan-IIA on Mia PaCa-2 cells. AKT, protein kinase B; EGFR, epidermal growth factor receptor; ERK, extracellular signal-regulated kinase; IGF1R, insulin-like growth factor 1 receptor; MEK, mitogen-activated protein kinase kinase; mTOR, mammalian target of rapamycin; PI3K, phosphoinositide 3-kinase; PTEN, phosphatase and tensin homolog; VEGFR vascular endothelial growth factor receptor.

Raf (Fig. 3E and L) and MEK (Fig. 3F and L) in a time-dependent manner. However, the protein expression levels of ERK (Fig. 3G and L) and AKT (Fig. 3I and M) were significantly decreased only at 48 and $72 \mathrm{~h}$; the protein expression levels of VEGFR (Fig. 3C and L) and mTOR (Fig. 3J and M) were significantly decreased only at $72 \mathrm{~h}$; and PTEN (Fig. 3K and M) was only significantly decreased at $24 \mathrm{~h}$. Tan-IIA significantly decreased the protein expression levels of Ras (Fig. 3D and L) and PI3K (Fig. 3H and M) at 24,48 and $72 \mathrm{~h}$, but expression was slightly higher at $48 \mathrm{~h}$ than at 24 and $72 \mathrm{~h}$.

\section{Discussion}

Ras is the most frequently mutated oncogene in human cancer (13). ERK belongs to the mitogen-activated protein kinase (MAPK) pathway, and dysregulation of the Ras/Raf/MAPK signal pathway has been well documented in tumorigenesis (14-16). AKT has an important role in apoptosis and cell production. Furthermore, PI3K can stimulate cellular survival and proliferation, and is required for activation of AKT $(7,8)$. It is well known that the PI3K/Akt/mTOR pathway enables the proliferation and survival of cancer driven by growth factor receptors. The PI3K/Akt/mTOR pathway is another of the most frequently dysregulated kinase cascades in human cancer (9). Novel PI3K/AKT/mTOR pathway-targeting drugs can overcome everolimus resistance in pancreatic neuroendocrine tumors and cell lines (17). In pre-clinical and clinical studies, both Ras/Raf/MEK/ERK and PI3K/Akt/mTOR cascade inhibitors have been examined for possible uses (18-20). Natural products and their derivatives can overpower MAPK signaling and the PI3K pathways activated by oncogenic Ras (21).
The present results demonstrated that MiaPaCa- 2 cells treated with Tan-IIA exhibited decreased protein expression levels of EGFR, IGF1R, VEGFR, Ras, Raf, MEK, ERK, PI3K, AKT and mTOR. These findings indicated that one of the molecular mechanisms through which Tan-IIA inhibits MiaPaCa-2 cells may be via decreasing EGFR, IGFR and VEGFR expression, and inhibiting the Ras/Raf/MEK/ERK and PI3K/Akt/mTOR pathway cascades. To the best of our knowledge, the present study is the first to reveal that Tan-IIA may inhibit MiaPaCa-2 pancreatic cancer cells through decreasing the protein expression levels of EGFR, IGFR and VEGFR, and inducing a dual blockade of Ras/Raf/MEK/ERK and PI3K/Akt/mTOR pathways (Fig. 4). Although advances have been made in systemic therapies, the use of adjuvant treatment in pancreatic cancer remains uncertain; and the 5-year survival rate for patients with early stage pancreatic cancer remains $<25 \%$ (22). Further in vivo studies regarding the chemotherapeutic potential of Tan-IIA in human pancreatic cancer are required.

\section{Acknowledgements}

Not applicable.

\section{Funding}

The present study was supported by a grant from Changhua Christian Hospital (grant no. 103-CCHICO-003).

\section{Availability of data and materials}

All data generated or analyzed during this study are included in this published article. 


\section{Author contributions}

CCS was involved in designing, performing the experiments and manuscript submission.

\section{Ethics approval and consent to participate}

Not applicable.

\section{Patient consent for publication}

Not applicable.

\section{Competing interests}

The author declares that they have no competing interests.

\section{References}

1. Siegel RL, Miller KD and Jemal A: Cancer Statistics, 2016. Cancer J Clinical 66: 7-30, 2016.

2. Che AJ, Zhang JY, Li CH, Chen XF, Hu ZD and Chen XG: Separation and determination of active components in Radix Salviae miltiorrhizae and its medicinal preparations by nonaqueous capillary electrophoresis. J Sep Sci 27: 569-575, 2004

3. Zhou L, Zuo Z and Chow MS: Danshen: An overview of its chemistry, pharmacology, pharmacokinetics, and clinical use. J Clin Pharmacol 45: 1345-1359, 2005.

4. Conroy T, Desseigne F, Ychou M, Bouché O, Guimbaud R, Bécouarn Y, Adenis A, Raoul JL, Gourgou-Bourgade S, de la Fouchardière $\mathrm{C}$, et al: FOLFIRINOX versus gemcitabine for metastatic pancreatic cancer. N Engl J Med 364: 1817-1825, 2011.

5. Yuen JS and Macaulay VM: Targeting the type 1 insulin-like growth factor receptor as a treatment for cancer. Expert Opin Ther Targets 12: 589-603, 2008.

6. Chitnis MM, Yuea JS, Protheroe AS, Pollak M and Macaulay VM: The type 1 insulin-like growth factor receptor pathway. Clin Cancer Res 14: 6364-6370, 2008.

7. Santarpia L, Lippman SM and El-Naggar AK: Targeting the MAPK-RAS-RAF signaling pathway in cancer therapy. Expert Opin Ther Targets 16: 103-119, 2012.

8. Liu P, Cheng H, Roberts TM and Zhao JJ: Targeting the phosphoinositide 3-kinase pathway in cancer. Nat Rev Drug Discov 8: 627-644, 2009.
9. McCubrey JA, Steelman LS, Kempf CR, Chappell WH, Abrams SL, Stivala F, Malaponte G, Nicoletti F, Libra M, Basecke J, et al: Therapeutic resistance resulting from mutations in Raf/MEK/ERK and PI3K/PTEN/Akt/mTOR signaling pathways. J Cell Physiol 226: 2762-2781, 2011.

10. Huang CY, Chiu TL, Kuo SJ, Chien SY, Chen DR and Su CC: Tanshinone IIA inhibits the growth of pancreatic cancer BxPC-3 cells by decreasing protein expression of TCTP, MCL-1 and Bcl-xL. Mol Med Rep 7: 1045-1049, 2013.

11. Su CC: Tanshinone IIA could inhibit pancreatic cancer BxPC-3 cells through increasing PERK, ATF6, caspase-12 and CHOP expression to induce apoptosis. J Biomedical Sci Engineering 8: 149-159, 2015.

12. Fronza M, Murillo R, Ślusarczyk S, Adams M, Hamburger M, Heinzmann B, Laufer S and Merfort I: In vitro cytotoxic activity of abietane diterpenes from Peltodon longipes as well as Salvia miltiorrhiza and Salvia sahendica. Bioorg Med Chem 19: 4876-4881, 2011.

13. Kranenburgm O, Gebbink MF and Voest EE: Stimulation of angiogenesis by Ras proteins. Biochim Biophys Acta 1654: 23-37, 2004.

14. Boonstra J, Rijken P, Humbel B, Cremers F, Verkleij A and van Bergen en Henegouwen P: The epidermal growth factor. Cell Biol Int 19: 413-430, 1995.

15. Cary LA, Han DC and Guan JL: Integrin-mediated signal transduction pathways. Histol Histopathol 14: 1001-1009, 1999.

16. Stacey DW: Cyclin D1 serves as a cell cycle regulatory switch in actively proliferating cells. Curr Opin Cell Biol 15: 158-163, 2003.

17. Vandamme T, Beyens M, de Beeck KO, Dogan F, van Koetsveld PM, Pauwels P, Mortier G, Vangestel C, de Herder W, Van Camp G, et al: Long-term acquired everolimus resistance in pancreatic neuroendocrine tumours can be overcome with novel PI3K-AKT-mTOR inhibitors. Br J Cancer 114: 650-658, 2016

18. McCubrey JA, Steelman LS, Chappell WH, Abrams SL, Franklin RA, Montalto G, Cervello M, Nicoletti F, Fagone P, Malaponte G, et al: Mutations and deregulation of Ras/Raf/MEK/ERK and PI3K/PTEN/Akt/mTOR cascades which alter therapy response. Oncotarget 3: 954-987, 2012.

19. Sebolt-Leopold JS: Advances in the development of cancer therapeutics directed against the Ras-mitogen-activated protein kinase pathway. Clin Cancer Res 14: 3651-3656, 2008.

20. Haura EB, Ricart AD, Larson TG, Stella PJ, Bazhenova L, Miller VA, Cohen RB, Eisenberg PD, Selaru P, Wilner KD, et al: A phase II study of PD-0325901, an oral MEK inhibitor, in previously treated patients with advanced non-small cell lung cancer. Clin Cancer Res 16: 2450-2457, 2010.

21. Quah SY, Tan MS, Teh YH and Stanslas J: Pharmacological modulation of oncogenic Ras by natural products and their derivatives: Renewed hope in the discovery of novel anti-Ras drugs. Pharmacol Ther 162: 35-57, 2016.

22. Goodman MD and Saif MW: Adjuvant therapy for pancreatic cancer. JOP 15: 87-90, 2014. 